\title{
Multidisciplinary Management of Acromegaly: A Consensus
}

Andrea Giustina $^{1}$, Garni Barkhoudarian ${ }^{2}$, Albert Beckers ${ }^{3}$, Anat Ben-Shlomo ${ }^{4}$, Nienke

Biermasz $^{5}$, Beverly Biller ${ }^{6}$, Cesar Boguszewski ${ }^{7}$, Marek Bolanowski ${ }^{8}$, Jens Bollerslev ${ }^{9}$, Vivien

Bonert $^{4}$, Marcello D. Bronstein ${ }^{10}$, Michael Buchfelder ${ }^{11}$, Felipe Casanueva ${ }^{12}$, Philippe

Chanson $^{13}$, David Clemmons ${ }^{14}$, Maria Fleseriu ${ }^{15}$, Anna Maria Formenti ${ }^{1}$, Pamela Freda ${ }^{16,}$

Monica Gadelha ${ }^{17}$, Eliza Geer ${ }^{18}$, Mark Gurnell ${ }^{19}$, Anthony P. Heaney ${ }^{20}$, Ken K.Y. Ho, ${ }^{21}$ Adriana

G. Ioachimescu ${ }^{22}$, Steven Lamberts ${ }^{23}$, Edward Laws ${ }^{24}$, Marco Losa ${ }^{25}$, Pietro Maffei ${ }^{26}$, Adam

Mamelak $^{4}$, Moises Mercado ${ }^{27}$, Mark Molitch $^{28}$, Pietro Mortini ${ }^{25}$, Alberto M. Pereira ${ }^{5}$, Stephan

Petersenn $^{29}$, Kalmon Post ${ }^{30}$, Manuel Puig-Domingo ${ }^{31}$, Roberto Salvatori ${ }^{32}$, Susan L. Samson ${ }^{33}$,

Ilan Shimon ${ }^{34}$, Christian Strasburger ${ }^{35}$, Brooke Swearingen ${ }^{36}$, Peter Trainer $^{37}$, Mary L.Vance ${ }^{38}$,

John Wass ${ }^{39}$, Margaret E. Wierman ${ }^{40}$, Kevin C.J. Yuen ${ }^{41}$ Maria Chiara Zatelli $^{42}$, Shlomo

Melmed $^{4}$

${ }^{1}$ Institute of Endocrine and Metabolic Sciences, San Raffaele Vita-Salute University and IRCCS

San Raffaele Hospital, Milan, Italy

${ }^{2}$ Pacific Pituitary Disorders Center, Pacific Neuroscience Institute, Santa Monica, California,

USA

${ }^{3}$ Department of Endocrinology, University of Liège, Liège, Belgium

${ }^{4}$ Pituitary Center, Cedars-Sinai Medical Center, Los Angeles, California, USA

${ }^{5}$ Division of Endocrinology and Center for Endocrine Tumors, Department of Medicine, Leiden

University Medical Center, Leiden, The Netherlands 
${ }^{6}$ Neuroendocrine Unit, Massachusetts General Hospital, Harvard Medical School, Boston, Massachusetts, USA

${ }^{7}$ SEMPR, Endocrine Division, Department of Internal Medicine, Federal University of Parana, Curitiba, Brazil

${ }^{8}$ Department of Endocrinology, Diabetes and Isotope Therapy, Wroclaw Medical University, Wroclaw, Poland

${ }^{9}$ Section of Specialized Endocrinology, Department of Endocrinology, University of Oslo, Oslo, Norway

${ }^{10}$ Neuroendocrine Unit, Division of Endocrinology and Metabolism, Hospital das Clinicas, University of Sao Paulo, Sao Paulo, Brazil

${ }^{11}$ Department of Neurosurgery, University Hospital Erlangen, Erlangen, Germany

${ }^{12}$ Division of Endocrinology, Santiago de Compostela University and Ciber OBN, Santiago de Compostela, Spain

${ }^{13}$ Assistance Publique-Hôpitaux de Paris, Hôpital Bicêtre, Service d'Endocrinologie et des Maladies de la Reproduction, Centre de Référence des Maladies Rares de l'Hypophyse and Université Paris-Saclay, Univ. Paris-Sud, Inserm, Signalisation Hormonale, Physiopathologie Endocrinienne et Métabolique, Le Kremlin-Bicêtre, Paris, France

${ }^{14}$ Department of Medicine, University of North Carolina, Chapel Hill, North Carolina, USA

${ }^{15}$ Departments of Medicine and Neurological Surgery, Pituitary Center, Oregon Health \& Science University, Portland, Oregon, USA

${ }^{16}$ Department of Medicine, Columbia University, Vagelos College of Physicians and Surgeons, New York, New York, USA 
${ }^{17}$ Neuroendocrinology Research Center/Endocrinology Section, Medical School and Hospital Universitário Clementino Fraga Filho, Universidade Federal do Rio de Janeiro, Rio de Janeiro, Brazil

${ }^{18}$ Multidisciplinary Pituitary and Skull Base Tumor Center, Memorial Sloan-Kettering Cancer Center, New York, New York, USA

${ }^{19}$ Metabolic Research Laboratories, Wellcome Trust-MRC Institute of Metabolic Science, University of Cambridge and NIHR Cambridge Biomedical Research Centre, Addenbrooke's Hospital, Cambridge, United Kingdom

${ }^{20}$ Division of Endocrinology, Diabetes and Hypertension, Department of Medicine, David Geffen School of Medicine, University of California, Los Angeles, California, USA

${ }^{21}$ The Garvan Institute of Medical Research, St. Vincent's Hospital and the University of New South Wales, Sydney, Australia

${ }^{22}$ Department of Neurosurgery and Medicine, Division of Endocrinology, Metabolism and Lipids, and Department of Neurosurgery, Emory University School of Medicine, Atlanta, Georgia, USA

${ }^{23}$ Erasmus Medical Center, Rotterdam, The Netherlands

${ }^{24}$ Pituitary/Neuroendocrine Center, Brigham \& Women's Hospital, Boston, Massachusetts, USA

${ }^{25}$ Department of Neurosurgery, San Raffaele University Health Institute Milan, Milan, Italy

${ }^{26}$ Department of Medicine, Padua University Hospital, Padua, Italy

${ }^{27}$ Medical Research Unit in Endcrine Diseases, Hospital de Especialidades, Centro Médico

Nacional, Siglo XXI, IMSS, Facultad de Medicina, Universidad Nacional Autónoma de México, Mexico City, Mexico 
${ }^{28}$ Division of Endocrinology, Metabolism \& Molecular Medicine, Northwestern University

Feinberg School of Medicine, Chicago, Illinois, USA

${ }^{29}$ ENDOC Center for Endocrine Tumors, Hamburg, Germany

${ }^{30}$ Department of Neurosurgery, Professor of Neurosurgery \& Medicine, Mount Sinai Health System, New York, New York, USA

${ }^{31}$ Germans Trias i Pujol Research Institute, Universitat Autònoma de Barcelona, Badalona, Barcelona, Spain

${ }^{32}$ Division of Endocrinology, Diabetes, and Metabolism and Pituitary Center, Johns Hopkins School of Medicine, Baltimore, USA

${ }^{33}$ Departments of Medicine and Neurosurgery, Baylor St. Luke's Pituitary Center, Baylor College of Medicine, Houson, Texas, USA

${ }^{34}$ Endocrine Institute, Rabin Medical Center, Beilinson Hospital, Petah-Tikva, Israel

${ }^{35}$ Department of Medicine for Endocrinology, Diabetes and Nutritional Medicine, Charité Universitätsmedizin, Berlin, Germany

${ }^{36}$ Department of Neurosurgery, Massachusetts General Hospital, Boston, Massachusetts, USA

${ }^{37}$ Department of Endocrinology, The Christie NHS Foundation Trust, University of Manchester, Manchester Academic Health Science Centre, Manchester, United Kingdom

${ }^{38}$ Department of Medicine, University of Virginia, Charlottesville, Virginia, USA

${ }^{39}$ Department of Endocrinology, Oxford Centre for Diabetes, Endocrinology and Metabolism, Churchill Hospital, Oxford, United Kingdom

${ }^{40}$ Pituitary, Adrenal and Neuroendocrine Tumor Program, Department of Medicine, University of Colorado Anschutz Medical Center, Aurora, Colorado, USA 
${ }^{41}$ Barrow Pituitary Center and Neuroendocrinology Clinic, Barrow Neurological Institute, University of Arizona College of Medicine and Creighton School of Medicine, Phoenix, Arizona, USA

${ }^{42}$ Section of Endocrinology \& Internal Medicine, Department of Medical Sciences, University of Ferrara, Ferrara, Italy

\section{Corresponding author:}

Prof. Andrea Giustina

Chair of Endocrinology

Ospedale San Raffaele

Via Olgettina, 58

20132 - Milano, Italy

e-mail: giustina.andrea@hsr.it 


\begin{abstract}
The 13th Acromegaly Consensus Conference was held in November 2019 in Fort Lauderdale, Florida, and comprised acromegaly experts including endocrinologists and neurosurgeons who considered optimal approaches for multidisciplinary acromegaly management. Focused discussions reviewed techniques, results, and side effects of surgery, radiotherapy, and medical therapy, and how advances in technology and novel techniques have changed the way these modalities are used alone or in combination. Effects of treatment on patient outcomes were considered, along with strategies for optimizing and personalizing therapeutic approaches. Expert consensus recommendations emphasize how best to implement available treatment options as part of a multidisciplinary approach at Pituitary Tumor Centers of Excellence.
\end{abstract}

\title{
Word count 3011
}

Keywords: acromegaly, consensus, multidisciplinary management, medical therapy, surgery, radiotherapy, Pituitary Tumor Centers of Excellence 


\section{Introduction}

Acromegaly is a chronic, progressive, and potentially lethal disease caused by a growth hormone (GH)-secreting pituitary adenoma and resultant excess in circulating levels of GH and insulinlike growth factor (IGF)-I (1). Facial and acral changes due to soft tissue overgrowth as well as systemic complications affecting bone and joints (2) and the cardio-respiratory system (3), in association with metabolic and oncologic complications, contribute to an increased clinical burden, leading to decreased quality of life and diminished survival rates $(4,5)$. Unfortunately, most patients already exhibit features of advanced disease at presentation due to a delay in diagnosis from first symptom onset by up to 8-10 years (6). Treatment of acromegaly is targeted to normalizing biochemical parameters as well as improving well being, controlling signs and symptoms, and reducing excess morbidity and mortality $(7,8)$. A multimodal therapeutic approach comprising neurosurgery, medical therapy, and radiotherapy is often required to attain these goals (9). Therefore, a multidisciplinary team approach is recommended for effective management of acromegaly and its comorbidities, coordinated by pituitary medicine experts to personalize treatment and follow-up, and optimize outcomes (10).

In November 2019, the Acromegaly Consensus Group convened in Fort Lauderdale, Florida, to provide current consensus on the comprehensive multidisciplinary management of acromegaly. Forty-eight acromegaly experts including endocrinologists and neurosurgeons reviewed the current literature and assessed current treatment choices and prioritization for clinical practice. Discussions focused on treatment outcome goals; results and side effects of neurosurgery, radiotherapy, and medical therapy; and the proposed place of each available treatment option in the guidelines. Updated consensus recommendations on treatment of patients with acromegaly were graded using the Grading of Recommendations Assessment, Development 
and Evaluation (GRADE) system (11). Key recommendations are presented in Table 1 and outlined in Figure 1.

\section{Methods}

Literature searches were performed by meeting participants to identify new data in English language papers published between January 2014 and October 2019, and indexed in PubMed. Search terms included "acromegaly" and terms associated with each topic, including “biochemical control”, "tumor volume”, "clinical symptoms”, "side effects”, "neurosurgery”, "radiotherapy", "somatostatin analogue", "somatostatin receptor ligand", "pegvisomant", “morbidity”, "mortality", "quality of life", and "guidelines". After brief plenary overviews on the state of the art for each topic, participants were divided into breakout groups for further analysis of the assigned topics and subsequently reported their conclusions to the whole group.

Consensus recommendations were produced based on speaker presentations, subgroup discussions, and reports. After the meeting, the Scientific Committee graded the evidence supporting the recommendations, and then graded the consensus recommendations on the basis of the quality of evidence (Table 2). Final graded consensus recommendations were approved by all meeting participants.

\section{Targets for Therapeutic Approaches}

\section{GH and IGF-I}

Excess GH and/or IGF-I lead to systemic comorbidities in patients with acromegaly, requiring effective treatment to decrease disease burden and reduce or normalize excess mortality (HQ) (12). Although consideration of tumor and clinical variables is important for clinical 
management, biochemical control is the cornerstone on which succesful treatment is built .Thus, at present, normalization of GH and IGF-1 is still the primary goal of acromegaly treatment and biochemical parameters should be used to evaluate activity of disease (SR).

GH nadir $<1 \mu \mathrm{g} / \mathrm{L}$ after an oral glucose tolerance test (OGTT) was initially defined by our Consensus Group as a marker of postsurgical remission (13); subsequently, this recommendation was revised to $0.4 \mu \mathrm{g} / \mathrm{L}$ taking into account use of ultrasensitive $\mathrm{GH}$ assays (14). However, $\mathrm{GH}$ nadir levels during an OGTT are impacted by factors such as patient age, BMI, sex, and estrogen status (15) (LQ), as well as glucose intolerance and diabetes mellitus or preexisting use of antidiabetic and somatostatin receptor ligand (SRL) therapy (VLQ) (16). Nevertheless, as these cutpoints correlate well with long-term outcomes (17), we recommend that ultrasensitive assays be used for diagnosis, and post-surgical evaluation using the $0.4 \mu \mathrm{g} / \mathrm{L}$ threshold for cut-off (SR).

During follow-up, IGF-I levels reflect clinical activity of disease (MQ) (1). However, wide variability between assays has been reported due to several preanalytical and analytical confounding factors (MQ) (18), and fluctuation of circulating IGF-I levels may be seen, particularly in the early postoperative period or after treatment changes (MQ) (19). It is therefore recommended that the same well-validated IGF-I assay be used throughout patient follow-up (SR). Further, although the absolute cut-off for defining biochemical control is the upper limit of normal (ULN) (SR), values slightly higher than this cut-off (e.g., within 1.2-1.3 × ULN) could be considered as a target of treatment depending on the clinical scenario $(20,21)$ (DR). Serum GH values can be used to assess control, with the goal of achieving a fasting level $<1.0 \mu \mathrm{g} / \mathrm{L}$. Close follow-up is recommended for patients with discrepant GH and IGF-1 levels observed at 3 months postoperatively; most commonly, patients show controlled GH and elevated IGF-I, but 
the opposite may also occur $(22,23)$. In these cases, we recommend relying on IGF-I values (SR).

\section{Tumor Volume}

Tumor growth control, and ideally, decreasing tumor size, are clinically important goals for patients with acromegaly (SR) (4). We recommend to continue evaluating reduction in mass maximal dimension, rather than overall tumor volume, which is not standardized (24) (DR). As the latter is a better measure of response, a consensus on methodology for measuring tumor volume would be welcomed by the physician community. T2-weighted MRI hypointensity may be helpful for predicting SRL therapy responsiveness (MQ) (25-27), along with adenoma granularity and other histological markers (VLQ) (7), but are not currently validated for guiding treatment. Tumor characteristics, such as the degree of adenoma fibrosis and consistency may be evaluated by texture analysis which is currently restricted to clinical trial settings to evaluate clinical precision.

\section{Clinical Symptoms}

As symptoms and comorbidities associated with acromegaly impact quality of life and survival, their prevention and control is a major goal of treatment (SR) (4). We recommend assessing and aggressively managing disease-associated comorbidities (SR). However, symptoms and clinical manifestations can be dissociated from biochemical values (LQ) (28), and specific assessment and clinical monitoring is recommended beyond biochemical parameters (SR). 
Clinician-reported instruments such as SAGIT (Signs and symptoms, Associated comorbidities, GH levels, IGF1 levels and Tumour profile) (29) and ACRODAT (Acromegaly Disease Activity Tool) (30) as well as patient-reported outcome assessment measures have been proposed to standardize follow-up over time (VLQ) (31-33), and their use can be considered in therapeutic decision-making (DR).

\section{Neurosurgery}

\section{Techniques}

Tumor resection via transsphenoidal surgery is the optimal primary treatment in most patients (HQ) (34) (Figure 1). Data supporting use of endoscopic over microscopic approaches remain incomplete and further comparative outcome studies are needed before one approach can be recommended over the other. Currently, the choice of technique depends on neurosurgeon expertise and preference. Craniotomy is very rarely indicated in patients with acromegaly (HQ) (35). Intraoperative MRI and other techniques to aid in intraoperative visualization of tumor remnants remain investigational (LQ) $(36,37)$.

\section{Results}

The primary predictor of the likelihood of achieving surgical remission remains tumor size and invasiveness of surrounding structures, particularly the cavernous sinus (HQ) $(38,39)$. Knosp grading may be correlated to outcomes (40). Preoperative serum GH level is also an important determinant of surgical remission $(41,42)$.

In specialized referral centers, remission can be achieved in $80-90 \%$ of microadenomas and about $50 \%$ to $75 \%$ of macroadenomas, although these figures dramatically decrease when 
the tumor is invasive or very large (e.g., $>4 \mathrm{~cm}$ ). (HQ) $(43,44)$. Remission rates are likely lower at less experienced centers

Surgical tumor debulking prior to medical therapy can be considered in appropriate candidates if the patient cannot be surgically cured (MQ), if a substantial amount of the mass can be successfully removed and/or there are symptoms of mass effect (45). Debulking may also be appropriate prior to radiotherapy to decrease target volume (DR).

Serum IGF-I levels to reliably define remission should be assessed at least 3 months postoperatively $(\mathrm{HQ})(43,44)$. Early indication of remission may be obtained by measuring fasting $\mathrm{GH}$ on postoperative day 1 or 2 , with lowest levels $(<1 \mu \mathrm{g} / \mathrm{L})$ having the best sensitivity to predict outcomes. However, these data need to be interpreted with caution if patients are treated with preoperative SRL therapy (VLQ) (43).

Expertise in surgical management of acromegaly, together with initial tumor dimension, has a dramatic impact on disease control rates (HQ) (10). A high volume of pituitary operations per individual surgeon per year with monitoring of outcome data is recommended to maintain sufficient surgical expertise (DR) (46).

\section{Preoperative SRL therapy}

Randomized studies suggest improvement in postoperative remission after pretreatment with SRL for 3-6 months (MQ). However, data are conflicting and, in many instances, results were not sustained during long-term follow-up (LQ) (47-50). The role of SRL pretreatment in improving anesthetic risk is not clear and current data do not support a general recommendation for preoperative SRL treatment (SR) (51). 


\section{Reoperations}

Reoperation may be considered in patients with significant residual tumor who have not adequately responded to postoperative SRL or in patients with a potentially resectable residual tumor after an unsuccessful first surgery (LQ) (52). Reoperation, as for primary surgery, should be done in a specialized center and after multidisciplinary evaluation (53) (SR).

\section{Complications}

Surgical complications after transsphenoidal surgery are well-recognized, although they occur less commonly with experienced surgeons (46). Post-surgical hypopituitarism can occur in 5$10 \%$ of cases and persistent CSF leakage in 2-3\%. (54) Other serious complications (e.g., visual deterioration, carotid artery injury, transient oculomotor palsies, and meningitis) are rarely observed (MQ) (55-57). Diabetes insipidus occurs at a rate similar to surgically treated pituitary tumors (10-15\%), and is usually transient. The syndrome of inappropriate antidiuretic hormone secretion may occur 5-14 days after surgery and requires vigilance, with frequent monitoring of serum sodium levels and possibly fluid restriction (LQ) $(58,59)$.

Advanced age, severe cardiomiopathy, and poorly controlled diabetes mellitus are relative contraindications to surgery (VLQ).

\section{Radiotherapy}

\section{Techniques}

Modern radiotherapy continues to have a place in the treatment algorithm, typically as a thirdline option after surgery and optimal medical therapy. There are two indications for radiotherapy: control of tumour growth and/or lowering GH secretion (60) (MQ). The earlier era of 
conventional radiotherapy was associated with complications, particularly cerebrovascular disease and secondary tumours, as well as hypopituitarism (9) (MQ). Modern stereotactic radiotherapy techniques are localised accurately in 3-dimensions, and are delivered either as a single fraction or fractionated. The relatively small number of patients undergoing pituitary radiotherapy and the long latency for an observed effect make it difficult to draw definitive conclusions about complication rates. However, single-fraction stereotactic radiosurgery appears to be associated with similar but fewer side effects as compared to fractionated radiotherapy (LQ) $(60-65)$.

Radiation therapy should be administered in specialized centers where patient selection is guided by discussion within a multidisciplinary team, and treatment should be delivered by radiotherapists experienced in treating pituitary disease to both maximize efficacy and prevent long-term complications (SR).

\section{Results}

Radiotherapy is reserved for patients that have failed, are unfit for, or declined surgical and/or medical therapy (SR) (Figure 1), and may be considered as second-line treatment in select patients (VLQ). Radiotherapy can control biochemical parameters in more than $60 \%$ of patients, and is highly efficacious $(>90 \%)$ in controlling tumor growth, offering the prospect of stopping high-cost lifelong medical therapy (MQ). However, full response may not be realized until up to 10-15 years after administration (MQ) (60-66). Given the delay in suppressing GH and IGF-I levels, medical therapy is indicated in the intervening years (SR).

\section{Side Effects and Contraindications}


Safety is the main limiting factor for use of radiation therapy in acromegaly, especially as safety of other treatment modalities has improved. There are currently no comparative studies of side effects caused by different modalities of radiotherapy. Reduced incidence of non-endocrine complications (i.e., secondary tumors, cerebrovascular disease, optic neuritis, cranial nerve palsy) may be observed with more focused techniques (LQ) (60-65). Hypopituitarism is the most frequent complication, regardless of technique, and increases over time, with rates approaching 25-50\% after 5 years (MQ) (67). Routine monitoring of endocrine function should be conducted lifelong (SR).

\section{Medical Therapy}

Medical therapy is recommended for patients who do not achieve biochemical control after surgery (SR). Primary medical therapy is reserved for those with contraindication to or who refuse surgery, and may be considered in select patients considered at poor risk for good outcomes and surgical success (DR) (68) (Figure 1).

\section{$S R L$}

Octreotide LAR and lanreotide are used as first-line medical therapy due to their favorable risk/benefit profiles (SR). Thirty to 55\% of patients achieve normal IGF-I on long-term treatment with these SRLs (MQ) (68-72) and >20\% reduction in tumor size is seen in more than half of treated patients (MQ) $(73,74)$. Lower baseline IGF-I level and older age are strong predictors of response (MQ) (75-77). Increasing dose and/or dose frequency of octreotide LAR and lanreotide can improve biochemical control rates in patients inadequately controlled on standard doses, but sensitive to SRL therapy (LQ) $(78,79)$. An oral formulation of octreotide was recently approved 
in the United States as long-term maintenance treatment in patients who have responded to and tolerated treatment with octreotide or lanreotide (80). Pasireotide LAR can be effective in normalizing IGF-1 levels in some patients inadequately controlled by octreotide LAR or lanreotide (MQ) (81-83), and may yield a higher rate of tumor shrinkage (LQ) (82).

Side effects of SRL include mainly gallstones and GI symptoms (1). Long-term octreotide LAR and lanreotide generally have an overall neutral effect on glucose metabolism (MQ), although in some patients mild hyperglycemia is observed (84). By contrast, pasireotide LAR causes hyperglycemia in up to $70 \%$ of patients, including secondary diabetes in $25-40 \%$ of patients (LQ) (85). Candidates for pasireotide LAR should therefore be carefully screened and monitored for glycemic adverse effects (SR). Controlled studies on the best treatment of pasireotide-induced hyperglycemia are not available. Patients not controlled on oral antidiabetic medications, including metformin, could be better managed with glucagon-like peptide-1 receptor agonists rather than insulin (DR) (86).

\section{Cabergoline}

Cabergoline, a relatively long acting dopamine agonist, has the advantages of limited cost and oral route of administration compared to SRL. However, its positioning in the therapeutic algorithm is limited by its relatively modest effect on inducing biochemical control, primarily restricted to patients who have mild-GH/IGF-I elevations postoperatively (IGF-I levels $<2.5 \mathrm{x}$ ULN) (7), as well as an escape phenomenon that can occur $(87,88)$. Some studies have suggested that cabergoline may be useful as add-on therapy in patients who do not achieve biochemical control with maximal doses of SRL (89) or pegvisomant (90) (VLQ) (DR). 


\section{Pegvisomant}

Unlike all other medical therapies, the $\mathrm{GH}$ receptor antagonist pegvisomant is not dependent on tumor characteristics for efficacy (91). Pegvisomant is generally used as second-line therapy in patients who do not achieve biochemical control with maximal doses of SRL (SR), although observational data suggest that it is also effective when used as first-line therapy (VLQ) (92). As higher rates of control are often seen as the dose is increased (93-97), treatment should be started at low doses and uptitrated as tolerated until control can be achieved (SR). Potentially, any patient can be controlled with adequate dose titration (MQ), but the high cost of treatment is often an obstacle to adequate dose titration (98) (VLQ).Younger patients with more aggressive disease, higher baseline IGF-I levels, and associated comorbidities may require higher doses to acheive biochemical control (LQ) (97). Loss of biochemical control due to tumor regrowth, previous treatment modifications, concomitant menopause, and changes in testosterone administration, can be corrected by increasing the dose (LQ) $(99,100)$.

Degree of improvement in clinical outcomes with pegvisomant is variable and is dependent upon the specific comorbidity and the duration of disease (101-103) (LQ). Compared to other forms of medical therapy, pegvisomant is the most likely to achieve maximal improvement in glucose tolerance and insulin sensitivity (MQ) (102, 103). Accordingly, pegvisomant is the preferred medical therapy for patients with preexisting hyperglycemia or diabetes mellitus who do not respond to octreotide LAR/lanreotide (SR). Abnormal liver function can occur early and should be monitored (SR) (97). Tumor size may rarely increase in patients switching from SRL, possibly as a rebound after stopping SRL but more likely due to the absence of a pituitary-targeting therapy $(97,104)$ (LQ). Pegvisomant is therefore preferred for patients with no clinically relevant residual tumor (SR). 


\section{Combination Pegvisomant and SRL Therapy}

Higher rates of biochemical control are seen in patients treated with combination pegvisomant and octreotide/lanreotide compared to those on SRL alone (MQ) $(104,105)$, and the combination may be considered in patients with a concern for residual tumor control and impaired glucose tolerance instead of switching to pasireotide LAR (DR) (8). The combination of pegvisomant and pasireotide LAR is effective in achieving biochemical control with lower pegvisomant doses but no clear advantage has yet been shown in attenuating the hyperglycemic effects of pasireotide (LQ) $(106,107)$. Nevertheless, this combination, although costly, may be an option among those with observed tumor growth if radiotherapy is either contraindicated or not available or while awaiting tumor-shrinking effects of radiation in more aggresive tumors (DR).

\section{Temozolomide}

Use of temozolomide and other chemotherapeutic agents should be limited to patients with highly aggressive or truly malignant pituitary tumors (108) and should be administered under supervision of a neuro-oncologist (109) (DR).

\section{The Multidisciplinary Treatment Approach}

The availability of increased management options has enabled a more effective multimodality treatment of acromegaly, requiring a higher degree of treatment personalization. Treatment of acromegaly is best determined by a multidisciplinary team of experts within the structure of a Pituitary Tumors Center of Excellence (PTCOE), preferably in a single institution where feasible (SR) (9) (MQ). The PTCOE should have a sufficiently large referral population to 
allow neurosurgeons to have post-residency training in a high-volume pituitary center, a continuous multidisciplinary experience, and a possibility to publish outcomes for pituitary tumor operations (DR) (46). Ideally, more than one surgeon per center should be available. In addition to experts in transsphenoidal pituitary surgery and pituitary disease endocrine management, the multidisciplinary team should include neuroradiologists, neuropathologists, radiation oncologists, and nurses with specific expertise in pituitary medicine (9) (LQ). A multidisciplinary treatment approach at a PTCOE where current guidelines are implemented and up-to-date and validated laboratory and clinical tools are routinely used offerrs the best opportunity for optimizing outcomes and quality of life while also ensuring that diseaseassociated morbidity and mortality are minimized (110) (SR). 


\section{Declarations}

\section{Ethical approval}

No original data from studies on patients are reported in this manuscript and therefore no ethical approval or informed consent was needed.

\section{Funding}

The 13th Acromegaly Consensus Conference was supported by unrestricted educational grants from Ipsen and Pfizer Inc. Scientific sponsorship of the meeting was provided by Cedars-Sinai Medical Center, Los Angeles, CA.

\section{Conflicts of Interest/Competing Interests}

AG is consultant for Chiasma, Genevant, Ipsen, Pfizer and Recordati and received research grants from Ipsen, Novartis and Pfizer to his Institution.

$\mathrm{AB}$ has received research funding from Pfizer and travel and speaker grants from Novartis and Ipsen

BMKB is PI of research support to Massachsuetts General Hospital from Crinetics, Ionis and Novartis, occasional consulting honoraria from Chiasma, Crinetics and Novartis.

CLB has served as consultant/speaker for Novartis and Ipsen.

MB has served as speaker for Novartis, Ipsen, Pfizer and Recordati

VB has received research grants from Pfizer, and has served on advisory boards for Pfizer MDB is Member of Steering Committees and Advisory Boards of Chiasma, Ipsen, Novartis; Speaker for Ipsen, Novartis; and has received grants as PI for clinical trials from Crinetics, Ipsen, and Novartis 
PC has received unrestricted research and educational grants from Ipsen, Novartis, Crinetics and Pfizer on behalf of the Service of Endocrinology and Reproductive Diseases, Hôpitaux Universitaires Paris-Saclay and Association Recherche Endocrinologie Bicêtre, and has served as investigator (principal or coordinator) for clinical trials funded by Novartis, Pfizer, Ipsen, Italpharmaco, Chiasma. PC gave lectures for Ipsen, Novartis, and Pfizer. All the fees and honoraria are paid to his Institution or Research Association.

DC served as an advisory board member for Crinetics

MF has received research support to OHSU as principal investigator from Chiasma, Crinetics, Ionis, Novartis and has received occasional scientific consulting from Chiasma, Crinetics, Ionis, Novartis, Pfizer.

PUF has received research grant to her institution for clinical research studies from Pfizer, Novartis, Ipsen and Chiasma.

MRG has been principal investigator in clinical trials from Novartis and Crinectics, has served on advisory boards for Novartis and Crinectics and served as apeaker for Novatis and Ipsen EBG is an investigator for research grants to MSKCC from Ionis, Novartis, Corcept, Strongbridge Biopharma, and Bristol-Myers Squibb. She has received a speaker's fee for an educational presentaion for Chiasma.

APH has served as consultant/ speaker for Ipsen and Chiasma AGI has received institution-directed research grants from Chiasma, Strongbridge and Novartis and served as consultant for Tiburio.

PM received consultation or speaker fee, support for congress participation, and contribution to research projects from by Pfizer, Novartis, and Ipsen MEM received research support from Chiasma, Novartis, Ionis, and Crinetics 
SP has served as an advisory board member for Ipsen, Novartis, and Crinetics, and as speaker for Ipsen, Novartis, and Pfizer.

MPD has recived research grants from Pfizer and Novartis, has served on advisory boards for Pfizer, Novartis and Ipsen, and served as speaker for Novartis, Ipsen and Pfizer.

RS received support for educational activities at Johns Hopkins University from Pfizer and support clinical trials from Chiasma, Crinetics and Novartis

SLS has served as an advisor and/or investigator for Novartis, Chiasma, Pfizer, and Ipsen, and has received investigator-initiated funding from Novartis.

IS has served as consultant and speaker for Novartis, Pfizer and Medison Pharma

CS served as an advisor to and received speaker's fees from Pfizer, Ipsen, Chiasma and Crinetics PT serves on advisory boards for Novartis, Crinetics, Chiasma and has received research funding to support clinical trials from Novartis, Crinetics, Chiasma, Strongbridge, Camarus. All honoraria and reseach income is administered by his institution.

MEW has received research grants to her institution for clinical research studies from Ionis and reviewed investigator-initiated research proposals for Pfizer.

KCJY has received research grants to his institution for clinical research studies from Ionis, Crinetics, and Novartis, and has served on advisory boards for Pfizer, and Ipsen. MCZ received consulting fees from Novartis and Ipsen.

SM has received consulting fees from Chiasma, Crinetics, Ionis, Ipsen and research grants to the institution from Pfizer.

GB, ABS, NB, JB, MB, FC, AMF, MG, KKYH, SL, EL, ML, AM, MM, PM, MP, KP, BS, MLV, and JW have no conflict of interests to declare 


\section{Authors' contributions}

AG and SM served as Program Coordinators of the 13th Acromegaly Consensus Conference. MB, FC, DC, MF, PM, SS, CS, and JW served as Scientific Committee Members. All authors researched data for the Conference. AG and SM wrote the manuscript and all authors reviewed and/or edited the manuscript before submission. 


\section{References}

1. Colao A, Grasso LFS, Giustina A, Melmed S, Chanson P, Pereira AM, Pivonello R. Acromegaly. Nat Rev Dis Primers. 2019;5:20.

2. Giustina A. Acromegaly and vertebral fractures: facts and questions. Trends Endocrinol Metab. 2020 Apr;31(4):274-275. doi: 10.1016/j.tem.2020.01.011. Epub 2020 Feb 1

3. Giustina A, Boni E, Romanelli G, Grassi V, Giustina G. Cardiopulmonary performance during exercise in acromegaly, and the effects of acute suppression of growth hormone hypersecretion with octreotide. Am J Cardiol. 1995;75(15):1042-1047. doi:10.1016/s00029149(99)80721-8

4. Giustina A, Barkan A, Beckers A, Biermasz N, Biller BMK, Boguszewski C, Bolanowski M, Bonert V, Bronstein MD, Casanueva FF, Clemmons D, Colao A, Ferone D, Fleseriu M, Frara S, Gadelha MR, Ghigo E, Gurnell M, Heaney AP, Ho K, Ioachimescu A, Katznelson L, Kelestimur F, Kopchick J, Krsek M, Lamberts S, Losa M, Luger A, Maffei P, Marazuela M, Mazziotti G, Mercado M, Mortini P, Neggers S, Pereira AM, Petersenn S, Puig-Domingo M, Salvatori R, Shimon I, Strasburger C, Tsagarakis S, van der Lely AJ, Wass J, Zatelli MC, Melmed S. A consensus on the diagnosis and treatment of acromegaly comorbidities: an update.J Clin Endocrinol Metab. 2020 Apr 1;105(4). pii: dgz096. doi: 10.1210/clinem/dgz096

5. Gadelha MR, Kasuki L, Lim DST, Fleseriu M. Systemic complications of acromegaly and the impact of the current treatment landscape: an update. Endocr Rev. 2019;40(1):268-332. doi:10.1210/er.2018-00115 
6. Petrossians P, Daly AF, Natchev E, et al. Acromegaly at diagnosis in 3173 patients from the Liège Acromegaly Survey (LAS) Database. Endocr Relat Cancer. 2017;24(10):505-518. doi:10.1530/ERC-17-

7. Melmed S Pituitary-Tumor Endocrinopathies. N Engl J Med. 2020 Mar 5;382(10):937-950.

8. Melmed S, Bronstein MD, Chanson P, et al A consensus statement on acromegaly therapeutic outcomes. Nat Rev Endocrinol. 2018;14:552-561.

9. Melmed S, Colao A, Barkan A, et al. Guidelines for acromegaly management: an update. $J$ Clin Endocrinol Metab. 2009;94(5):1509-1517. doi:10.1210/jc.2008-2421

10. Casanueva FF, Barkan AL, Buchfelder M, et al; Pituitary Society, Expert Group on Pituitary Tumors. Criteria for the definition of Pituitary Tumor Centers of Excellence (PTCOE): a Pituitary Society statement. Pituitary. 2017;20:489-498.

11. Guyatt G, Oxman AD, Akl EA, et al. GRADE guidelines: 1. Introduction-GRADE evidence profiles and summary of findings tables. J Clin Epidemiol. 2011;64(4):383-394. doi:10.1016/j.jclinepi.2010.04.026

12. Melmed S, Casanueva FF, Klibanski A, et al. A consensus on the diagnosis and treatment of acromegaly complications. Pituitary. 2013;16(3):294-302. doi:10.1007/s11102-0120420-X

13. Giustina A, Barkan A, Casanueva FF, et al. Criteria for cure of acromegaly: a consensus statement. J Clin Endocrinol Metab. 2000;85(2):526-529. doi:10.1210/jcem.85.2.6363

14. Giustina A, Chanson P, Bronstein MD, et al. A consensus on criteria for cure of acromegaly. J Clin Endocrinol Metab. 2010;95(7):3141-3148. doi:10.1210/jc.2009-2670 
15. Schilbach K, Gar C, Lechner A, et al. Determinants of the growth hormone nadir during oral glucose tolerance test in adults. Eur J Endocrinol. 2019;181(1):55-67. doi:10.1530/EJE-190139

16. Carmichael JD, Bonert VS, Mirocha JM, Melmed S. The utility of oral glucose tolerance testing for diagnosis and assessment of treatment outcomes in 166 patients with acromegaly. J Clin Endocrinol Metab. 2009;94(2):523-527. doi:10.1210/jc.2008-1371

17. Giustina A, Mazziotti G, Fontanella M. Commentary: Postsurgical monitoring of acromegaly. Neurosurgery. 2013;73(4):E746-E748. doi:10.1227/NEU.0000000000000068

18. Clemmons DR. IGF-I assays: current assay methodologies and their limitations. Pituitary. 2007;10(2):121-128. doi:10.1007/s11102-007-0032-Z

19. Feelders RA, Bidlingmaier M, Strasburger CJ, et al. Postoperative evaluation of patients with acromegaly: clinical significance and timing of oral glucose tolerance testing and measurement of (free) insulin-like growth factor I, acid-labile subunit, and growth hormone-binding protein levels. J Clin Endocrinol Metab. 2005;90(12):6480-6489. doi:10.1210/jc.2005-0901

20. Cunha MLVD, Borba LAB, Boguszewski CL. Random GH and IGF-I levels after transsphenoidal surgery for acromegaly: relation with long-term remission. Endocrine. 2020;68(1):182-191. doi:10.1007/s12020-020-02227-2

21. . Espinosa-de-los-Monteros AL, Gonzalez B, Vargas G, Sosa E, Mercado M. Octreotide, treatment of acromegaly in "real life": long-term outcome at a tertiary care center. Pituitary 18(3), 290-296 (2015). https://doi.org/10.1007/s11102-014-0570-0 
22. Bianchi A, Giustina A, Cimino V, et al. Influence of growth hormone receptor d3 and fulllength isoforms on biochemical treatment outcomes in acromegaly. J Clin Endocrinol Metab. 2009;94(6):2015-2022. doi:10.1210/jc.2008-1337

23. Schilbach K, Strasburger CJ, Bidlingmaier M. Biochemical investigations in diagnosis and follow up of acromegaly. Pituitary. 2017;20(1):33-45. doi:10.1007/s11102-017-0792-z

24. Amato G, Mazziotti G, Rotondi M, et al. Long-term effects of lanreotide SR and octreotide LAR on tumour shrinkage and GH hypersecretion in patients with previously untreated acromegaly. Clin Endocrinol (Oxf). 2002;56(1):65-71. doi:10.1046/j.03000664.2001.01438.x

25. Dogansen SC, Yalin GY, Tanrikulu S, et al. Clinicopathological significance of baseline T2-weighted signal intensity in functional pituitary adenomas. Pituitary. 2018;21:347-354.

26. Puig-Domingo M, Resmini E, Gomez-Anson B, et al. Magnetic resonance imaging as a predictor of response to somatostatin analogs in acromegaly after surgical failure. $J$ Clin Endocrinol Metab. 2010;95(11):4973-4978. doi:10.1210/jc.2010-0573

27. Potorac I, Beckers A, Bonneville JF. T2-weighted MRI signal intensity as a predictor of hormonal and tumoral responses to somatostatin receptor ligands in acromegaly: a perspective. Pituitary. 2017;20(1):116-120. doi:10.1007/s11102-017-0788-8

28. González B, Vargas G, de Los Monteros ALE, Mendoza V, Mercado M. Persistence of diabetes and hypertension after multimodal treatment of acromegaly. J Clin Endocrinol Metab. 2018;103(6):2369-2375. doi:10.1210/jc.2018-00325.

29. Giustina A, Bronstein MD, Chanson P, et al. Staging and managing patients with acromegaly in clinical practice: baseline data from the SAGIT validation study. Pituitary. 2019, 22: 476-487. 
30. van der Lely AJ, Gomez R, Pleil A, et al. Development of ACRODAT ${ }^{\circledR}$, a new software medical device to assess disease activity in patients with acromegaly. Pituitary. 2017;20(6):692-701. doi:10.1007/s11102-017-0835-5

31. Fleseriu M, Fogelfeld L, Gordon MB, et al. Development of a novel patient-reported measure for acromegaly: the Acro-TSQ. Pituitary. 2019;22:581-593.

32. Jackson Y, Flood E, Rhoten S, Janssen EM, Lundie M. AcroVoice: eliciting the patients' perspective on acromegaly disease activity. Pituitary. 2019;22:62-69.

33. Biermasz NR. The burden of disease for pituitary patients. Best Pract Res Clin Endocrinol Metab. 2019;33:101309.

34. Honegger J, Grimm F. The experience with transsphenoidal surgery and its importance to outcomes. Pituitary. 2018;21:545-555

35. Buchfelder M, Schlaffer SM, Zhao Y. The optimal surgical techniques for pituitary tumors. Best Pract Res Clin Endocrinol Metab. 2019;33:101299.

36. Amano K, Aihara Y, Tsuzuki S, Okada Y, Kawamata T. Application of indocyanine green flourescence endoscopic system in transsphenoidal surgery for pituitary tumors. Acta Neurochir. 2019;161:695-706.

37. Sandow N, Klene W, Elbelt U, Strasburger CJ, Vajkoczy P. Intraoperative indocyanine green videoangiography for identification of pituitary adenomas using a microscopic transsphenoidal approach. Pituitary. 2015;18(5):613-620. doi:10.1007/s11102-014-0620-7

38. Rutkowski M, Zada G. Management of pituitary adenomas invading the cavernous sinus. Neurosurg Clin N Am. 2019;30:445-455 
39. Antunes X, Ventura N, Camilo GB, et al. Predictors of surgical outcome and early criteria of remission in acromegaly. Endocrine. 2018;60(3):415-422. doi:10.1007/s12020-0181590-8

40. Micko AS, Wöhrer A, Wolfsberger S, Knosp E. Invasion of the cavernous sinus space in pituitary adenomas: endoscopic verification and its correlation with an MRI-based classification. J Neurosurg. 2015;122(4):803-811. doi:10.3171/2014.12.JNS141083

41. Jane JA, Jr., Starke RM, Elzoghby MA, et al. Endoscopic transsphenoidal surgery for acromegaly: remission using modern criteria, complications, and predictors of outcome. $\mathbf{J}$ Clin Endocrinol Metab 2011;96:2732-40

42. . Agrawal N, Ioachimescu AG. Prognostic factors of biochemical remission after transsphenoidal surgery for acromegaly: a structured review [published online ahead of print, 2020 Jun 29]. Pituitary. 2020;10.1007/s11102-020-01063-X

43. Mortini P, Barzaghi LR, Albano L, et al. Microsurgical therapy of pituitary adenomas. Endocrine. 2018;59:72-81.

44. Buttan A, Mamelak AN. Endocrine outcomes after pituitary surgery. Neurosurg Clin N Am. 2019;30:491-498.

45. Fahlbusch R, Kleinberg D, Biller B, et al. Surgical debulking of pituitary adenomas improves responsiveness to octreotide LAR in the treatment of acromegaly. Pituitary. 2017;20:668-675.

46. Mortini P, Nocera G, Roncelli F, Losa M, Formenti AM, Giustina A. The optimal numerosity of the referral population of pituitary tumors centers of excellence (PTCOE): A surgical perspective [published online ahead of print, 2020 Jun 2]. Rev Endocr Metab Disord. 2020;10.1007/s11154-020-09564-7. doi:10.1007/s11154-020-09564 
47. Albarel F, Castinetti F, Morange I, et al. Pre-surgical medical treatment, a major prognostic factor for long-term remission in acromegaly. Pituitary. 2018;21:615-623.

48. Polat Korkmaz O, Gurcan M, Nuhoglu Kantarci FE, Haliloglu O, Ozkaya HM, Sahin S, Oren MM, Tanriover N, Gazioglu N, Kadioglu P.. The effects of pre-operative somatostatin analogue therapy on treatment cost and remission in acromegaly. Pituitary. 2019;22:387-396.

49. Nunes VS, Correa JM, Puga ME, Silva EM, Boguszewski CL. Preoperative somatostatin analogues versus direct transsphenoidal surgery for newly-diagnosed acromegaly patients: a systematic review and meta-analysis using the GRADE system Pituitary. 2015 Aug; 18(4):500-8

50. Lv L, Hu Y, Zhou P, et al. Presurgical treatment with somatostatin analogues in growthhormone-secreting pituitary adenomas: a long-term single-center experience. Clin Neurol Neurosurg. 2018;167:24-30.

51. Losa M, Bollerslev J. Pros and cons in endocrine practice: pre-surgical treatment with somatostatin analogues in acromegaly. Endocrine. 2016;52:451-457.

52. Almeida JP, Ruiz-Treviño AS, et al. Reoperation for growth-hormone secreting pituitary adenomas: endonasal endoscopic series and systematic review of the literature. $J$ Neurosurg. 2018;129:404-416.

53. Espinosa de los Monteros AL, González B, Vargas G, Sosa E, Guinto G, Mercado M. Surgical reintervention in acromegaly: is it still worth trying? Endocr Pract. 2009;15:431437.

54. Chen CJ, Ironside N, Pomeraniec IJ, Chivukula S, Buell TJ, Ding D, Taylor DG, Dallapiazza RF, Lee CC, Bergsneider M. Microsurgical versus endoscopic transsphenoidal 
resection for acromegaly: a systematic review of outcomes and complications.Acta Neurochir (Wien). 2017 Nov;159(11):2193-2207. doi: 10.1007/s00701-017-3318-6.

55. Conger A, Zhao F, Wang X, et al. Evolution of the graded repair of CSF leaks and skull base defects in endonasal endoscopic tumor surgery: trends in repair failure and meningitis rates in 509 patients. J Neurosurg. 2018;130(3):861-875. doi:10.3171/2017.11.JNS172141.

56. Perry A, Graffeo CS, Meyer J, et al. Beyond the learning curve: comparison of microscopic and endoscopic incidences of internal carotid injury in a series of highly experienced operators. World Neurosurg. 2019;131:e128-e135.

57. Pagliano P, Caggiano C, Ascione T, et al. Characteristics of meningitis following transsphenoidal endoscopic surgery: a case series and a systematic literature review. Infection. 2017;45(6):841-848. doi:10.1007/s15010-017-1056-6

58. Bohl MA,Ahmad S, Jahnke H. Delayed hyponatremiais the most common cause of 30-day unplanned readmission after transsphenoidal surgery for pituitary tumors. Neurosurgery.2016 Jan;78(1):84-90.

59. Winograd D, Staggers KA, Sebastian S, Takashima M, Yoshor D, Samson SL. An effective and practical fluid restriction protocol to decrease the risk of hyponatremia and readmissions after transsphenoidal surgery [published online ahead of print, 2020 Jan 28]. Neurosurgery. 2020;nyz555. doi:10.1093/neuros/nyz555

60. Ding D, Mehta GU, Patibandla MR, et al. Stereotactic radiosurgery for acromegaly: an international multicenter retrospective cohort study. Neurosurgery. 2019;84:717-725.

61. Gheorghiu ML. Updates in outcomes of stereotactic radiation therapy in acromegaly. Pituitary. 2017;20:154-168. 
62. Gonzalez-Virla B, Vargas-Ortega G, Martínez-Vázquez KB, et al. Efficacy and safety of fractionated conformal radiation therapy in acromegaly: a long-term follow-up study. Endocrine. 2019;65:386-392.

63. Sala E, Moore JM, Amorin A, et al. CyberKnife robotic radiosurgery in the multimodal management of acromegaly patients with invasive macroadenoma: a single center's experience. J Neurooncol. 2018;138:291-298.

64. Weidlich GA, Bodduluri M, Achkire Y, Lee C, Adler JR Jr. Characterization of a novel 3 megavolt linear accelerator for dedicated intracranial stereotactic radiosurgery. Cureus. 2019;11(3):e4275.

65. Kong DS, Kim YH, Kim YH, et al. Long-term efficacy and tolerability of Gamma Knife radiosurgery for growth hormone-secreting adenoma: a retrospective multicenter study (MERGE-001). World Neurosurg. 2019;122:e1291-e1299.

66. Minniti G, Jaffrain-Rea ML, Osti M, et al. The long-term efficacy of conventional radiotherapy in patients with GH-secreting pituitary adenomas. Clin Endocrinol (Oxf). 2005;62(2):210-216. doi:10.1111/j.1365-2265.2005.02199.x

67. Cordeiro D, Xu Z, Li CE, et al. Hypopituitarism after Gamma Knife radiosurgery for pituitary adenomas: a multicenter international study. J Neurosurg. 2018;131:1188-1196.

68. Giustina A, Bronstein MD, Casanueva FF, et al. Current management practices for acromegaly: an international survey. Pituitary. 2011;14(2):125-133. doi:10.1007/s11102010-0269-9

69. Frara S, Maffezzoni F, Mazziotti G, Giustina A. The modern criteria for medical management of acromegaly. Prog Mol Biol Transl Sci. 2016;138:63-83. doi:10.1016/bs.pmbts.2015.10.015 
70. Alqurani H, Del Pilar Schneider M, Mirakhur B, Barkan A. Biochemical efficacy of longacting lanreotide depot/Autogel in patients with acromegaly naive to somatostatin-receptor ligands: analysis of three multicenter clinical trials. Pituitary. 2018;21:283-289.

71. Lamberts SW, Hofland L. ANNIVERSARY REVIEW: Octreotide, 40 years later. Eur J Endocrinol. 2019;181:R173-R183.

72. Colao A, Auriemma RS, Pivonello R, Kasuki L, Gadelha MR. Interpreting biochemical control response rates with first-generation somatostatin analogues in acromegaly.Pituitary. 2016 Jun;19(3):235-47. doi: 10.1007/s11102-015-0684-z.)

73. Giustina A, Mazziotti G, Torri V, Spinello M, Floriani I, Melmed S. Meta-analysis on the effects of octreotide on tumor mass in acromegaly. PLoS One. 2012;7(5):e36411. doi:10.1371/journal.pone.0036411

74. Mazziotti G, Giustina A. Effects of lanreotide SR and Autogel on tumor mass in patients with acromegaly: a systematic review. Pituitary. 2010;13(1):60-67. doi:10.1007/s11102009-0169-z

75. Coopmans EC, Korevaar TIM, van Meyel SWF, et al. Multivariable prediction model for biochemical response to first-generation somatostatin receptor ligands in acromegaly. $\mathrm{J}$ Clin Endocrinol Metab. 2020 Jun 26;dgaa387. doi: 10.1210/clinem/dgaa387.

76. Caron PJ, Bevan JS, Petersenn S, et al. Tumor shrinkage with lanreotide Autogel $120 \mathrm{mg}$ as primary therapy in acromegaly: results of a prospective multicenter clinical trial. J Clin Endocrinol Metab. 2014;99(4):1282-1290. doi:10.1210/jc.2013-3318

77. Petersenn S, Houchard A, Sert C, Caron PJ; PRIMARYS Study Group. Predictive factors for responses to primary medical treatment with lanreotide autogel $120 \mathrm{mg}$ in acromegaly: 
post hoc analyses from the PRIMARYS study. Pituitary. 2020;23(2):171-181. doi:10.1007/s11102-019-01020-3

78. Giustina A, Bonadonna S, Bugari G, et al. High-dose intramuscular octreotide in patients with acromegaly inadequately controlled on conventional somatostatin analogue therapy: a randomised controlled trial. Eur J Endocrinol. 2009;161(2):331-338. doi:10.1530/EJE-090372

79. Giustina A, Mazziotti G, Cannavò S, et al. High-dose and high-frequency lanreotide autogel in acromegaly: a randomized, multicenter study. J Clin Endocrinol Metab. 2017;102(7):2454-2464. doi:10.1210/jc.2017-00142

80. Samson SL, Nachtigall LB, Fleseriu M, Gordon MB, Bolanowski M, Labadzhyan A et al. Maintenance of acromegaly control in patients switching from injectable somatostatin receptor ligands to oral octreotide therapy. J Clin Endocrinol Metab. 2020. doi:10.1210/clinem/dgaa526.

81. Colao A, Bronstein MD, Freda P, et al. Pasireotide versus octreotide in acromegaly: a head-to-head superiority study. J Clin Endocrinol Metab. 2014;99(3):791-799. doi:10.1210/jc.2013-2480

82. Gadelha MR, Bronstein MD, Brue T, et al. Pasireotide versus continued treatment with octreotide or lanreotide in patients with inadequately controlled acromegaly (PAOLA): a randomised, phase 3 trial. Lancet Diabetes Endocrinol. 2014;2(11):875-884. doi:10.1016/S2213-8587(14)70169-X

83. Colao A, Bronstein MD, Brue T, et al. Pasireotide for acromegaly: long-term outcomes from an extension to the Phase III PAOLA study. Eur J Endocrinol. 2020;182(6):583. doi:10.1530/EJE-19-0762 
84. Mazziotti G, Floriani I, Bonadonna S, Torri V, Chanson P, Giustina A. Effects of somatostatin analogs on glucose homeostasis: a metaanalysis of acromegaly studies. J Clin Endocrinol Metab. 2009;94(5):1500-1508. doi:10.1210/jc.2008-2332

85. Schmid HA, Brue T, Colao A, et al. Effect of pasireotide on glucose- and growth hormonerelated biomarkers in patients with inadequately controlled acromegaly. Endocrine. 2016;53(1):210-219. doi:10.1007/s12020-016-0895-8

86. Samson S, Gu F, Feldt-Rasmussen U, et al. A multicenter, randomized, open-label, phase IV study investigating management of pasireotide-associated hyperglycemia with incretinbased therapy or insulin in patients with acromegaly or Cushing's disease. J Endocr Soc. 2019;3(suppl 1):Abstract MON-LB079.

87. Howlett TA, Willis D, Walker G, et al. Control of growth hormone and IGF1 in patients with acromegaly in the UK: responses to medical treatment with somatostatin analogues and dopamine agonists. Clin Endocrinol (Oxf) 2013;79(5): 689-99.Clin Endocrinol (Oxf) 2018;88(6):889-95.

88. Sandret L, Maison P, Chanson P. Place of cabergoline in acromegaly: a metaanalysis.J Clin Endocrinol Metab 2011;96(5):1327-35.

89. Suda K, Inoshita N, Iguchi G, et al. Efficacy of combined octreotide and cabergoline treatment in patients with acromegaly: a retrospective clinical study and review of the literature. Endocr J 2013;60(4):507-15.

90. Bernabeu I, Alvarez-Escola C, Paniagua AE, et al. Pegvisomant and cabergoline combination therapy in acromegaly. Pituitary 2013;16(1):101-8. 
91. Giustina A, Arnaldi G, Bogazzi F, et al. Pegvisomant in acromegaly: an update [published correction appears in J Endocrinol Invest. 2017 Dec 28;:]. J Endocrinol Invest. 2017;40(6):577-589. doi:10.1007/s40618-017-0614-1

92. van der Lely AJ, Kuhn E, Muhammad A, Coopmans EC, Neggers SJ, Chanson P. Pegvisomant and not somatostatin receptor ligands (SRLs) is first-line medical therapy for acromegaly. Eur J Endocrinol. 2020;182(6):D17-D29. doi:10.1530/EJE-19-0998

93. Trainer PJ, Drake WM, Katznelson L, et al. Treatment of acromegaly with the growth hormone-receptor antagonist pegvisomant. N Engl J Med. 2000;342(16):1171-1177. doi:10.1056/NEJM200004203421604

94. van der Lely AJ, Hutson RK, Trainer PJ, et al. Long-term treatment of acromegaly with pegvisomant, a growth hormone receptor antagonist. Lancet. 2001;358(9295):1754-1759. doi:10.1016/s0140-6736(01)06844-1

95. Basavilbaso NXG, Ballarino MC, Bruera D, et al. Pegvisomant in acromegaly: a multicenter real-life study in Argentina. Arch Endocrinol Metab. 2019;63:320-327.

96. Boguszewski CL, Huayllas MKP, Vilar L, et al. Brazilian multicenter study on pegvisomant treatment in acromegaly. Arch Endocrinol Metab. 2019;63:328-336.

97. Buchfelder M, van der Lely AJ, Biller BMK, et al. Long-term treatment with pegvisomant: observations from 2090 acromegaly patients in ACROSTUDY. Eur J Endocrinol. 2018;179(6):419-427. doi:10.1530/EJE-18-0616

98. Giustina A. Optimal use of pegvisomant in acromegaly: are we getting there?. Endocrine. 2015;48(1):3-8. doi:10.1007/s12020-014-0462-0 
99. Franck SE, Korevaar TIM, Petrossians P, et al. A multivariable prediction model for pegvisomant dosing: monotherapy and in combination with long-acting somatostatin analogues. Eur J Endocrinol. 2017;176(4):421-431. doi:10.1530/EJE-16-0956

100. van der Lely AJ, Jönsson P, Wilton P, Åkerblad AC, Cara J, Ghigo E. Treatment with high doses of pegvisomant in 56 patients with acromegaly: experience from ACROSTUDY. Eur J Endocrinol. 2016;175(4):239-245. doi:10.1530/EJE-16-0008

101. Chiloiro S, Giampietro A, Frara S, et al. Effects of pegvisomant and pasireotide LAR on vertebral fractures in acromegaly resistant to first-generation SRLs. J Clin Endocrinol Metab. 2020;105(3):dgz054. doi:10.1210/clinem/dgz054

102. Brue T, Lindberg A, van der Lely A, et al. Diabetes in patients with acromegaly treated with pegvisomant: observations from ACROSTUDY. Endocrine. 2019;63:563-572.

103. Feola T, Cozzolino A, Simonelli I, et al. Pegvisomant improves glucose metabolism in acromegaly: a meta-analysis of prospective interventional studies. J Clin Endocrinol Metab. 2019;104:2892-2902.

104. Strasburger CJ, Mattson A, Wilton P, Aydin F, Hey-Hadavi J, Biller BMK. Increasing frequency of combination medical therapy in the treatment of acromegaly with the GH receptor antagonist pegvisomant. Eur J Endocrinol. 2018;178:321-329.

105. Leonart LP, Tonin FS, Ferreira VL, Fernandez-Llimos F, Pontarolo R. Effectiveness and safety of pegvisomant: a systematic review and meta-analysis of observational longitudinal studies. Endocrine. 2019;63:18-26.

106. Muhammad A, van der Lely AJ, Delhanty PJD, et al. Efficacy and safety of switching to pasireotide in patients with acromegaly controlled with pegvisomant and first-generation 
somatostatin analogues (PAPE Study). J Clin Endocrinol Metab. 2018;103(2):586-595. doi:10.1210/jc.2017-02017.

107. Chiloiro S, Bima C, Tartaglione T, et al. Pasireotide and pegvisomant combination treatment in acromegaly resistant to second line therapies: a longitudinal study. J Clin Endocrinol Metab. 2019;104:5478-5482

108. Ho KKY, Fleseriu M, Wass J, et al. A tale of pituitary adenomas: to NET or not to NET: Pituitary Society position statement. Pituitary. 2019;22:569-573.

109. Whitelaw BC. How and when to use temozolomide to treat aggressive pituitary tumours. Endocr Relat Cancer. 2019;26(9):R545-R552. doi:10.1530/ERC-19-0083

110. Frara S, Rodriguez-Carnero G, Formenti AM, Martinez-Olmos MA, Giustina A, Casanueva FF Pituitary Tumors Centers of Excellence Endocrinol Metab Clin N Am published online July 152020

\section{Table 1. Key Recommendations*}

1. During follow-up, measurement of IGF-I levels with the same well-validated assay is recommended. Values slightly higher than a standard cut-off for age-adjusted normalization (e.g., within 1.2-1.3 $\times$ ULN) may be considered sufficient for control of acromegaly.

2. Prevention and control of symptoms and comorbidities is a major goal of treatment. Assessing and aggressively managing disease-associated comorbidities is recommended, with use of clinician- and patient-reported outcome measures to help standardization of follow-up strategies. 
3. Tumor resection via transsphenoidal surgery (either endocscopic or microscopic) is a safe and effective primary treatment for most patients. The primary predictors of surgical remission are tumor size, invasiveness (Knosp grade), and experience of the neurosurgeon.

4. Medical therapy is recommended for patients who do not achieve biochemical control after surgery. Choice of therapy among dopamine agonist, SRL, and GH receptor antagonist should be individualized based on disease- and patient-specific factors known to affect therapeutic efficacy and safety.

5. Radiotherapy is reserved for patients that have failed, are unfit for, or declined surgical and/or medical therapy. It should be administered in specialized centers to maximize efficacy and minimize long-term complications

6. Treatment of acromegaly is best determined by a multidisciplinary team of experts within the structure of a PTCOE, preferably in a single institution with a sufficiently large referral population. Such an approach is more likely to optimize outcomes and quality of life while minimizing disease-associated morbidity and decreasing mortality.

*These recommendations were selected among all the recommendations included in the text based on a formal vote from all authors and reflect the consensus reached within the group. 


\section{Table 2. Grading of Evidence and Recommendations}

\begin{tabular}{|l|l|}
\hline Grading the evidence & Grading the recommendations \\
\hline $\begin{array}{l}\text { Supported by one or few small } \\
\text { uncontrolled studies }\end{array}$ & • based on VLQ or LQ evidence \\
Low quality (LQ): supported by large & MQ or HQ evidence \\
series of small uncontrolled studies & \\
Moderate quality (MQ): supported by one & \\
or few large uncontrolled studies or meta- & \\
analyses & \\
High quality (HQ): supported by & \\
controlled studies or large series of large & \\
uncontrolled studies with sufficiently long & \\
follow-up
\end{tabular}

Adapted with permission from Melmed et al (7) 


\section{Figure 1. Algorithm for the Multidisciplinary Management of Acromegaly}

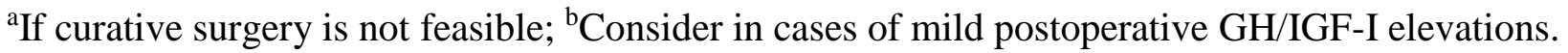
Well controlled defined as normalized GH/IGF-I; not controlled defined as other than wellcontrolled. Abbreviations: IGF-I, insulin-like growth factor- I; SRL, somatostatin receptor ligand octreotide or lanreotide. 
